mass and the ordinate being the required damping in the tank. It is assumed that 16 percent of the spacecraft's mass is sloshing at the location of the slosh mass. Values $\gamma_{s}$ above the curve indicate the stable region. It can be seen that bending increases the danger zone and requires larger damping of the propellant. Furthermore, the effect of angle-of-attack meter and accelerometer control with $g_{2}=1 / g$ is clearly expressed in the respective curves.

The potential hazard in control due to propellant sloshing in space vehicles can be eliminated by proper choice of tank form (slosh mass ratio decrease and slosh frequency increase), proper selection of type, location, and gain values of control sensors, and - as a last resort-by baffles.

\section{Critical Frequencies in the Stagnation Region of a Shock Layer $\dagger$}

\section{R. J. Leite and N. E. Hawk*}

Radiation Laboratory, Department of Electrical Engineering, University of Michigan, Ann Arbor, Mich.

January 9, 1961

$\mathbf{T}$ O FACILITATE the estimating of stagnation-region plasma and electron, neutral-particle collision frequencies, a plot of altitude versus velocity with equilibrium plasma and collision frequencies indicated parametrically was developed. Since the gas in the stagnation region is relatively dense and only weaklyionized, a so-called critical frequency is introduced to account for the influence of these elastic collisions. Alteration of the plasma frequency, when collisions are considered, is indicated on the above-mentioned plot.

The term "plasma," as used here, refers to an electrically neutral, ionized gas consisting of equal populations of electrons and ions, and neutral particles also. The assumption of electrical neutrality implies that the effects of space-charge forces, namely internally generated electric fields, are not important at distances comparable to the shortest length of interest. In the case of electromagnetic radiation, this length is the wavelength of the radiation considered. It is well known that a characteristic frequency, called the plasma frequency, $\omega_{p}$, is associated with each plasma, namely,

$$
\omega_{p}^{2}=n e^{2} / m \epsilon
$$

where $n$ is the electron number density, $e$ is the charge of an electron, $m$ is its mass and $\epsilon$ is the dielectric constant of free space. Physically, $\omega_{p}$ can be thought of as the frequency at which electrons will oscillate about their equilibrium positions after being displaced by an external force. Upon being displaced, the electrons are subjected to a restoring force due to the electric field between them and the ions, which move very little because of their greater mass. This restoring force will tend to return the electrons to their equilibrium positions and will cause them to oscillate, in an undamped manner, about these positions, in the absence of a magnetic field. It can be shown that the influence of free electrons is always to reduce the dielectric constant below the free-space value, namely,

$$
\epsilon^{\prime}=\epsilon\left[1-\left(\omega_{p} / \omega\right)^{2}\right]
$$

where $\epsilon^{\prime}$ is the effective dielectric constant of the plasma and $\omega$ is the frequency of an incident electromagnetic wave.

Determination of electron density in the stagnation region of

† This research was supported by the Advanced Research Projects Agency and the U.S. Army Signal Research and Development Agency under Con tract No. DA 36-039 SC-75041, ARPA Order No. 120-60. Project Code No. 7700 .

* Research Engineer and Assistant in Research, respectively. the shock layer during re-entry requires reference to some model atmosphere. ${ }^{1}$ In addition, real-gas effects must be considered in the evaluation of properties ${ }^{2}$ and electronic states ${ }^{3}$ of the air in that region. In Ref. 2 the Rankine-Hugoniot shock-wave equations are solved with dissociation and ionization included. Using these shock-wave results and the data in Ref. 3, the equilibrium electron density as a function of velocity and altitude can be computed easily. Using the relationship

$$
f_{p}=8.97 \sqrt{ } \bar{n}
$$

where $f_{p}=\omega_{p} / 2 \pi$ is the plasma frequency in cps and $n$ is the number of electrons per cubic meter, loci of constant plasma frequency are drawn on the altitude-velocity plot shown in Fig. 1. Superposition of any altitude-velocity trajectory on this plot will indicate the plasma frequency in the stagnation region at points along the path of the re-entry body.

In general, a plasma will reflect electromagnetic energy which is radiated at frequencies less than $f_{p}$ and it will absorb this energy when the radiating frequency is equal to or greater than $f_{p}$. At frequencies only slightly greater than $f_{p}$, attenuation of the incident electromagnetic wave is quite large; however, as the propagation frequency becomes increasingly greater than $f_{p}$ the effect of the free electrons becomes less pronounced and conditions approach those of free space. This can be readily seen by referring to Eq. (2), which shows that $\epsilon$ is approached as propagation frequencies become greate: than $f_{p}$.

The above would be true in a collision-free gas. In the shock layer however, the gas density is of such magnitude that freeelectron, neutral-particle collisions must be considered during at least part of the re-entry flight. Collisions between free electrons and positive ions can be disregarded, however, because of the degree of ionization is usually small - of the order of several percent. Hence, the above discussion of the propagation of electromagnetic waves in the stagnation region must be modified when the electron, neutral-particle collision frequency, $\nu$, begins to approach $f_{p}$. When this happens, the classical definition of plasma frequency [Eq. (1)] will remain unchanged, although its physical significance becomes doubtful. In other words the ordered motion of the electrons tends to be made random by the electron, neutral-particle collision processes and this, in turn, influences the electromagnetic properties of the plasma.

Referring to Whitmer, ${ }^{4}$ we see that $\epsilon^{\prime}$ of a plasma in which collisions are considered is

$$
\epsilon^{\prime}=\epsilon\left[1-\frac{\omega_{p}^{2}}{\nu^{2}+\omega^{2}}+i \frac{\omega_{p}^{2}(\nu / \omega)}{\nu^{2}+\omega^{2}}\right]
$$

where $\nu$ is collisions per second. The inclusion of $\nu$ has resulted

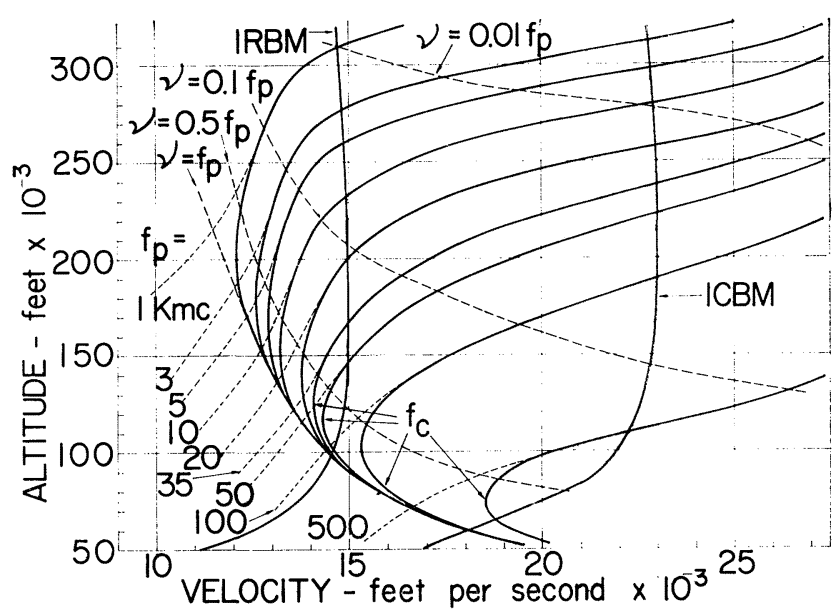

Frg. 1. Plasma, collision, and critical frequencies present in the stagnation region of the shock layer of a re-entry vehicle. $\left(2 \pi f_{p}\right)^{2}=\left(n e^{2} / m \epsilon\right)$;

$$
\nu=3.6 \times 10^{11} \rho_{2} / \rho_{0}\left(T_{2} / 1,000\right)^{1 / 2}, f_{c}^{2}=f_{p}{ }^{2}\left[1-\left(\nu / \omega_{p}\right)^{2}\right]
$$

Typical trajectories ${ }^{5}$ of several types of missiles are also shown. 
in a complex value of $\epsilon^{\prime}$, thereby indicating that the plasma now has some additional loss properties which further attenuate any propagating electromagnetic wave. In addition, it can be shown that the electron velocity is no longer $90^{\circ}$ out of phase with the electric field of the incident electromagnetic wave as it was in the collision-free case. This means that the free electrons can now absorb energy from the incident electric field, thereby increasing the degree of ionization of the gas because of the occurrence of higher-energy interactions during electron, neutral-particle collisions.

Eq. (2) shows that in the collision-free case the critical frequency of the gas (that frequency at which absorption is maximum) is equal to $\omega_{p}$. When collisions are considered, Eq. (4) indicates that the critical frequency of the gas is dependent upon both $\omega_{p}$ and $\nu$. This is seen readily by setting the real part of Eq. (4) equal to zero, namely,

$$
\omega_{c}^{2}=\omega_{p}^{2}\left[1-\left(\nu / \omega_{p}\right)^{2}\right]
$$

where $\omega_{c}$ is the critical frequency of the gas with collisions. We see that when $\nu$ is small compared with $\omega_{p}$, Eq. (5) yields results for the collision-free case.

Eq. (5) has been evaluated in the shock layer and the results are shown in Fig. 1, where $f_{c}=\omega_{c} / 2 \pi$. Collision frequency was determined from the relationship

$$
\nu=3.6 \times 10^{11} \rho_{2} / \rho_{0}\left(T_{2} / 1,000\right)^{1 / 2}
$$

where $\rho_{2}$ and $T_{2}$ are stagnation-region values of density and temperature, respectively, and $\rho_{0}$ is standard sea-level density. It should be noted that when $\nu$ is less than 10 percent of $f_{p}$, the propagation conditions of a collision-free gas may be assumed for all practical purposes. On the other hand for greater values of $\nu$, the $f_{c}$ of the gas decreases with increasing $\nu$, thereby permitting additional frequencies, namely $\omega_{p}>\omega>\omega_{c}$, to propagate through the gas. In the limit, when $\nu=\omega_{p}$, at least in principle, all frequencies propagate through the gas with some degree of attenuation.

\section{REFERENCES}

1 Minzner, R. A., Champion, K, S. W, and Pond, H. L, The ARDC Model Atmosphere, 1959, Geophysics Research Directorate, AFCRC, ARDC, Air Force Surveys in Geophysics, No. 115, AFCRC-TC-TC-59-267, August 1959.

${ }^{2}$ Hochstim, A. R., Gas Properties Behind Shocks at Hypersonic Velocities, I. Normal Shocks in Air, Convair, San Diego, Physics Group, Rep. No. $\mathrm{ZPh}(\mathrm{GP})-002$, Jan. 30, 1957.

3 Gilmore, F. R., Equilibrium Composition and Thermodynamic Properties of Air to $24,000^{\circ} \mathrm{K}$, The RAND Corporation, RM-1543, Aug. 24, 1955.

${ }^{4}$ Whitmer, R. F., Principles of Microwave Interactions with Ionized Media, The Microwave Journal, Vol. 2, No. 2, pp. 17-19, Feb. 1959.

5 Rose, P. H., and Stark, W. I., Stagnation Point Heat-Transfer Measurements in Dissociated Air, Journal of the Aeronautical Sciences, Vol. 25, No. 2, pp. 86-97, February 1958

\section{Analytical Expression for the Roll Rate of Aileron-Equipped Ballistic Re-Entry Bodies}

Martin R. Fink

Supervisor, Missile Aerodynamics Group,

United Aircraft Corporation Research Laboratories,

East Hartford, Conn.

March 3, 1961

\section{List of Symbols}

$=\mathrm{drag}$ reference area, $\mathrm{ft}^{2}$

$=$ reference length, $\mathrm{ft}$

$=$ drag coefficient

$C_{l}(\delta)=$ rolling-moment coefficient caused by ailerons

$C_{l p}=$ damping-in-roll coefficient, $\partial C_{l} / \partial(\omega b / 2 \mathrm{~V})$

$I_{x x}=$ moment of inertia in roll, slug- $\mathrm{ft}^{2}$

$k_{0} \quad=$ drag parameter defined by Eq. (5)

$K \quad=$ damping parameter defined by Eq. (7)

$m \quad=$ mass of re-entry vehicle, slugs

$S \quad=$ rolling-moment reference area, $\mathrm{ft}^{2}$

$=$ time, sec

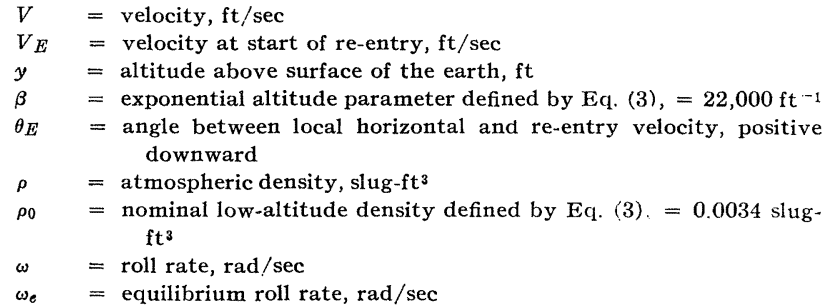

$\mathbf{B}$ ALLISTIC re-entry bodies may require an angular velocity about the longitudinal axis to minimize dispersion caused by inadequate static stability and nonzero trimmed lift coefficient. A rolling velocity can be imparted either by aileron surfaces affixed to the body, which produce a roll rate which increases during re-entry, or by reaction jets, which may be heavier and less reliable than fixed aileron surfaces but which can produce the desired roll rate prior to re-entry. In order to evaluate these two methods, an analytical expression is required for the variation of roll rate with altitude for ballistic re-entry bodies which utilize aileron-induced roll. Such a relation is derived herein from analytical studies of ballistic re-entry trajectories.

The angular acceleration in roll, $d \omega / d t$, is determined by the equation

$$
I_{x x}(d \omega / d t)=C_{l}(\delta)(\rho / 2) V^{2} S b+C_{l_{p}}(\rho / 2) V^{2} S b(\omega b / 2 V)
$$

where $C_{l}(\delta)$ is the rolling-moment coefficient due to control-panel deflection and $C_{l_{p}}$ is the damping-in-roll coefficient. The trajectory of a typical nonlifting ballistic re-entry body is essentially linear at the altitudes at which roll is developed, and the re-entry trajectory analysis given in Ref. 1 can be utilized. Thus, the rate of change of altitude with time can be approximated by

$$
d y / d t=-V \sin \theta_{E}
$$

and the variation of atmospheric density with altitude can be approximated by

$$
\rho=\rho_{0} e^{-\beta y}
$$

From Eq. (13) of Ref. 1

$$
V=V_{E} e^{-\frac{k_{0}}{2} e^{-\beta y}}
$$

where

$$
k_{0}=\left(\rho_{0} / \beta \sin \theta_{E}\right)\left(C_{D} A / m\right)
$$

Eq. (1) can then be rearranged as

$$
\frac{d \omega}{d(-\beta y)}+K e^{-\beta y} \omega=C_{l}(\delta) \frac{S b}{I_{x x}} \frac{k_{0} V_{E}}{2} \frac{m}{C_{D} A} e^{-\beta y} e^{-\frac{k_{0}}{2} e^{-\beta y}}
$$

where

$$
K=-C_{l_{p}}\left(S b^{2} / I_{x x}\right)\left(\rho_{0} / 4 \beta \sin \theta_{E}\right)
$$

The aerodynamic coefficients are assumed constant and the roll rate is assumed equal to zero prior to re-entry. Eq. (6) can then be integrated to give

$$
\omega=C_{l}(\delta) \frac{S b}{I_{x x}} V_{E} \frac{m}{C_{D} A} \frac{k_{0}}{k_{0}+2 K}\left(e-K e^{-\beta y}-e^{-\frac{k_{0}}{2} e^{e-\beta y}}\right)
$$

in $\mathrm{rad} / \mathrm{sec}$, which reduces to

$$
\omega=C_{l}(\delta)\left(S b / I_{x x}\right) V_{E}\left(m / C_{D} A\right)\left(1-e^{-\frac{k_{i}}{2} e-\beta y}\right)
$$

if the damping-in-roll coefficient, $C_{l_{p}}$, is assumed equal to zero.

The calculated variation of roll rate with altitude is tabulated below for a representative ICBM re-entry body which has an average roll control moment per unit dynamic pressure, $C_{l}(\delta) S b$, of 1 in. $^{3}$. This value of roll control moment includes a large loss of control effectiveness caused by immersion of the panels within the boundary layer. The tabulated values of roll are based on a moment of inertia, $I_{x x}$, of 15 slug- $\mathrm{ft}^{2}$, a ballistic coefficient, $m g / C_{D} A$, of $1,000 \mathrm{psf}$, a minimum-energy $5,500 \mathrm{~nm}$ trajectory, and damping in roll such that $K / k_{0}$ is equal to zero (no damping in roll) and 0.1 . For this case, the roll rate increases 